\title{
ON THE MAXIMAL NUMBER OF NON-OVERLAPPING KLEIN 4-GROUPS INSIDE AN ELEMENTARY ABELIAN 2-GROUP
}

\author{
DAVID E. DOBBS
}

\begin{abstract}
Let $G$ be a finite elementary abelian 2-group of order $2^{n}$, for some integer $n \geq 2$. Let $b_{n}$ be the maximal cardinality of a set $\mathcal{S}$ of subgroups of $G$ such that each member of $\mathcal{S}$ is isomorphic to the Klein 4-group and any two distinct members of $\mathcal{S}$ meet only in 0 . It is proved that $b_{n+2} \geq 4 b_{n}$. Consequently, $b_{n} \geq 2^{n-2}$ if $n$ is even, while $b_{n} \geq 2^{n-3}$ if $n$ is odd; these results are best possible since $b_{2}=1=b_{3}$.
\end{abstract}

\section{Introduction}

On June 17, 2008, Jim Coykendall telephoned me to ask for help in proving a conjecture that had arisen in his ongoing collaboration with Sean Sather-Wagstaff on fan graphs. To state the conjecture, we need the following notation and terminology. Let $G$ be a finite elementary abelian 2-group of order $2^{n}$, for some integer $n \geq 2$; that is, $G$ is a direct sum of $n$ copies of $\mathbb{Z} / 2 \mathbb{Z}$. Distinct subgroups $H$ and $K$ of $G$ are said to be non-overlapping if $H \cap K=0$. Let $b_{n}$ denote the maximal cardinality of a set $\mathcal{S}$ of pairwise non-overlapping subgroups of $G$ such that each member of $\mathcal{S}$ is isomorphic to the Klein 4-group $V(:=\mathbb{Z} / 2 \mathbb{Z} \oplus \mathbb{Z} / 2 \mathbb{Z})$. The conjecture states that $b_{n} \geq 2^{n-2}-2$. Later that day, I emailed Coykendall my proof that $b_{n} \geq 2^{n-2}$ if $n$ is even, while $b_{n} \geq 2^{n-3}$ if $n$ is odd. I added that these results are best possible since $b_{2}=1=b_{3}$. In particular, the original conjecture failed if $n=3$; and the bound for $b_{n}$ that I had proved for even $n$ was sharper than the bound in the original conjecture. Three days later, Coykendall replied that he had intended the original conjecture only for $n \geq 4$ and, for that context, he had just finished proving the original conjecture, adding that this would be enough for the graph-theoretic applications that he and Sather-Wagstaff envisaged. Given that our methods were different and our results complemented one another, we agreed to publish our work separately. This note combines the substance of my email message of June 17, 2008 (see Theorem 1 and Corollary 2 below) and a calculation sharpening the bound for $b_{5}$ that I made a few days later (see Remark $3(\mathrm{~b})$ ).

Received October 6, 2008.

2000 Mathematics Subject Classification. Primary 20K01; Secondary 05A99.

Key words and phrases. Elementary abelian 2-group, nonoverlapping subgroups, Klein 4-group, fan graph, partial spread. 
After the work on this note had been completed, I learned of some relevant work [1] which was motivated, according to its authors, "by the design of noncoherent multipleantenna communication systems". In [1, Corollary III.3], the estimation of $b_{n}$ was, in effect, reinterpreted by viewing two-dimensional $\mathbb{F}_{2}$-subspaces of $\mathbb{F}_{2} \oplus \mathbb{F}_{2}$ as lines in an associated projective space. One consequence of [1, Corollary III.3] is that if $n$ is even, then $b_{n}=\left(2^{n}-1\right) / 3$. For even $n \geq 4$, this result is sharper than Corollary 2 (a) below (and hence sharper than the result of Coykendall and Sather-Wagstaff). For odd $n$, the authors of [1] refer readers to some computer-aided searches involving partial $t$-spreads. After learning of [1], I have concluded that there are several reasons to publish the work that is being reported on here. First, it is algorithmic: see the proof of Theorem 1, as well as parts (b) and (c) of Remark 3. Second, the key step (Theorem 1) does not depend on the parity of $n$. Third, in view of [1, Corollary III.3], we see that for even $n$, the factor 4 in the statement of Theorem 1 is of the right order of magnitude, as the best-possible factor would be

$$
\frac{2^{n+2}-1}{2^{n}-1}=4+\frac{3}{2^{n}-1}
$$

Fourth, our methods are more elementary, in the sense that they avoid any consideration of projective geometry.

\section{Results}

Theorem 1. If $n \geq 2$ is an integer, then $b_{n+2} \geq 4 b_{n}$.

Proof. It will be convenient to consider $G$ as the external direct sum of $n+2$ copies of $\mathbb{Z} / 2 \mathbb{Z}$. Let $U$ denote the direct sum of the the first $n$ copies of $\mathbb{Z} / 2 \mathbb{Z}$. As the direct sum of the last two copies of $\mathbb{Z} / 2 \mathbb{Z}$ can be identified with the Klein 4 -group $V$, we have $G=U \oplus V$. It suffices to prove that if $\mathcal{S}$ is a set of cardinality $N$ consisting of pairwise non-overlapping subgroups of $U$ which are each isomorphic to $V$, then one can construct a set $\mathcal{T}$ of cardinality $4 N$ consisting of pairwise non-overlapping subgroups of $G$ which are each isomorphic to $V$. We shall do this by showing that each $W=\left\{w_{1}, w_{2}, w_{3}, 0\right\} \in \mathcal{S}$ leads to four elements of the eventual set $\mathcal{T}$. As we view $U \subseteq G$ in the usual way, we have $W \subseteq G$, and so it is natural to take the first of the members of $\mathcal{T}$ that arise from $W$ to be $W$ itself.

To define the other three members of $\mathcal{T}$ that arise from $W$, we need the following notation. Let the elements of $V$ be denoted by $a, b, c, 0$. Note that $2 a=2 b=2 c=0$, while $a+b=c, a+c=b$, and $b+c=a$. Consider the following three subsets of $G$ :

$$
X:=\left\{w_{1}+a, w_{2}+b, w_{3}+c, 0\right\}, Y:=\left\{w_{1}+b, w_{2}+c, w_{3}+a, 0\right\},
$$

and $Z:=\left\{w_{1}+c, w_{2}+a, w_{3}+b, 0\right\}$. Note that $2 w_{1}=2 w_{2}=2 w_{3}=0$, while $w_{1}+w_{2}=$ $w_{3}, w_{1}+w_{3}=w_{2}$, and $w_{2}+w_{3}=w_{1}$. It is straightforward to check that $X, Y$ and $Z$ are subgroups of $G$ that are each isomorphic to $V$. For instance, to check that $X$ has cardinality 4 , one would need to know, in particular, that $w_{1}+a \neq w_{2}+b$, and this 
follows since $w_{1}-w_{2}=w_{3} \in W \backslash\{0\}$ and $b-a=c \in V \backslash\{0\}$. (One should perhaps add that the last two coordinates of $w_{3}$ are each 0 , while this is not the case for $c$.) Using similar reasoning, one can check that $W, X, Y$ and $Z$ are pairwise non-overlapping.

It remains to show that the result of constructing four non-overlapping subgroups of $G$ (each of which is isomorphic to $V$ ) via each of the $N$ members of $\mathcal{S}$ produces $4 N$ non-overlapping subgroups of $G$. Consider any nonzero element $g=\left(g_{1}, \ldots, g_{n+2}\right)$ of one of these subgroups. Note that all the elements of members of $\mathbb{S}$ have their last two coordinates equal to 0 ; and that all the elements of $V$ have (when viewed in $G$ ) their first $n$ coordinates equal to 0 . Thus, $\left(g_{1}, \ldots, g_{n}\right)$ is either 0 or an element of a uniquely determined member, say $W$, of $\mathcal{S}$ (since the members of $\mathcal{S}$ are pairwise non-overlapping); and $\left(g_{1}, g_{2}\right)$ is a uniquely determined member of $V$. By knowing which element of $W$ has been added to which element of $V$ to produce $g$, we then know exactly which one of the sets $W, X, Y, Z$ contains $g$. This completes the proof.

Corollary 2. Consider any integer $n \geq 2$. Then:

(a) If $n$ is even, then $b_{n} \geq 2^{n-2}$.

(b) If $n$ is odd, then $b_{n} \geq 2^{n-3}$.

Proof. It is clear from the definition of $b_{n}$ that $b_{2}=1$. To see that $b_{3}=1$, consider any isomorphic copy $H$ of the Klein 4-group which is a subgroup of $G=\mathbb{Z} / 2 \mathbb{Z} \oplus$ $\mathbb{Z} / 2 \mathbb{Z} \oplus \mathbb{Z} / 2 \mathbb{Z}$. As $H$ is an internal direct summand of $G$ (by, for instance, vector space considerations), we may take $H=V=\mathbb{Z} / 2 \mathbb{Z} \oplus \mathbb{Z} / 2 \mathbb{Z}$ and $G=H \oplus \mathbb{Z} / 2 \mathbb{Z}$. Writing the elements of $\mathbb{Z} / 2 \mathbb{Z}$ as 0 and 1 , note that there do not exist three distinct elements of $G$ of the form $\left(z_{1}, z_{2}, 1\right)$ such that these elements, together with the zero element of $G$, constitute a subgroup of $G$. Hence, there does not exist a subgroup $K \neq H$ of $G$ such that $K \cong V$ and $H \cap K=0$. Thus $b_{3} \leq 1$, with the reverse inequality holding by virtue of $H$. This proves that $b_{3}=1$. In view of Theorem 1 , the assertions in (a) and (b) now follow easily by induction.

Remark 3. (a) As we saw in the proof of Corollary 2 that $b_{2}=1$ (resp., $b_{3}=1$ ), it follows that Corollary 2 (a) (resp., Corollary 2 (b)) is best possible.

(b) Corollary 2 (b) yields that $b_{5} \geq 4$, while the result announced by Cokendall and Sather-Wagstaff yields that $b_{5} \geq 2^{5-2}-2=6$. One can do better, as we show next that $b_{5} \geq 7$.

We shall produce a set $\left\{W, X, Y, Z, N_{1}, N_{2}, N_{3}\right\}$ of seven pairwise distinct pairwise non-overlapping subgroups of $G$ (viewed as the external direct sum of five copies of $\mathbb{Z} / 2 \mathbb{Z}$ ) which are each isomorphic to the Klein 4-group. The method of discovery is hinted at in (c), and we leave to the reader the task of verifying that the set which is 
given below does have the asserted properties. The relevant definitions are as follows:

and

$$
\begin{aligned}
W & :=\{(0,0,0,0,0),(0,1,0,0,0),(1,0,0,0,0),(1,1,0,0,0)\} \\
X & :=\{(0,0,0,0,0),(0,1,0,1,0),(1,0,1,0,0),(1,1,1,1,0)\} ; \\
Y & :=\{(0,0,0,0,0),(0,1,1,0,0),(1,0,1,1,0),(1,1,0,1,0)\} ; \\
Z & :=\{(0,0,0,0,0),(0,1,1,1,0),(1,0,0,1,0),(1,1,1,0,0)\} ; \\
N_{1} & :=\{(0,0,0,0,0),(0,1,0,0,1),(0,1,0,1,1),(0,0,0,1,0)\} ; \\
N_{2} & :=\{(0,0,0,0,0),(1,0,0,0,1),(1,0,1,0,1),(0,0,1,0,0)\} ;
\end{aligned}
$$

$$
N_{3}:=\{(0,0,0,0,0),(0,0,1,1,1),(0,0,0,0,1),(0,0,1,1,0)\} .
$$

(c) It is natural to ask if $b_{5} \geq 8$, as it would then follow (by reasoning as in the proof of Corollary 2) that $b_{n} \geq 2^{n-2}$ for all $n \geq 2$. A laborious case analysis (which is left to the reader) shows that the only impediment to proving that $b_{5} \geq 8$ arises if one has four non-overlapping copies of the Klein 4-group, say $W=\left\{w_{1}, w_{2}, w_{3}, 0\right\}, X=$ $\left\{x_{1}, x_{2}, x_{3}, 0\right\}, Y$ and $Z$, inside $\mathbb{Z} / 2 \mathbb{Z} \oplus \mathbb{Z} / 2 \mathbb{Z} \oplus \mathbb{Z} / 2 \mathbb{Z} \oplus \mathbb{Z} / 2 \mathbb{Z}$ such that for each $w_{i} \in W$, there exist a unique index $j(i)$ such that $w_{i}+x_{j(i)} \notin Y \cup Z$ and $\{j(1), j(2), j(3)\}=$ $\{1,2,3\}$. Unfortunately, if one finds non-overlapping $W, X, Y, Z$ by applying the reasoning of Theorem 1 and Corollary 2 (a) to get that $b_{4}=4$ and then augments $\{W, X, Y, Z\}$ as in the construction in the second paragraph of (b), one can check that the resulting set $\left\{W, X, Y, Z, N_{1}, N_{2}, N_{3}\right\}$ does satisfy the above-noted "impediment". However, a computer search should easily settle the question of whether $b_{5} \geq 8$ (or $b_{7} \geq 32$ or $b_{9} \geq 128$ or $\left.\cdots\right)$. If any such search successfully finds an odd $\nu \geq 5$ such that $b_{\nu} \geq 2^{\nu-2}$, then Corollary 2 could be strengthened to say that $b_{n} \geq 2^{n-2}$ for all $n \geq \nu$.

(d) Apart from the specific question raised in (c), other related questions remain open. For instance, one could replace $\mathbb{Z} / 2 \mathbb{Z}$ with $\mathbb{Z} / m \mathbb{Z}$ for any integer $m \geq 2$ (or with any finite abelian group). We encourage the reader to pursue such generalizations and their possible graph-theoretic applications.

\section{References}

[1] F. E. Oggier, N. J. A. Sloane, S. N. Diggavi and A. R. Calderbank, Nonintersecting subspaces based on finite alphabets, IEEE Trans. Inform. Theory 51 (2005), 4320-4325.

Department of Mathematics, University of Tennessee, Knoxville, Tennessee 37996-1300, U.S.A.

E-mail: dobbs@math.utk.edu 